\title{
Impact of NPS MedicineWise General Practitioner education programs and Choosing Wisely Australia recommendations on prescribing of Proton Pump Inhibitors in Australia
}

Jianyun Wu

NPS MedicineWise

Scott Dickinson

NPS Medicinewise

Zain Elgebaly

NPS Medicinewise

Suzanne Gaye Blogg ( $\nabla$ sblogg@nps.org.au )

NPS Medicinewise https://orcid.org/0000-0001-8429-1922

Aine Heaney

NPS Medicinewise

Yien Soo

NPS Medicinewise

Benjamin Daniels

NPS Medicinewise

Lynn Weekes

NPS Medicinewise

Research article

Keywords:

Posted Date: August 20th, 2019

DOI: https://doi.org/10.21203/rs.2.13221/v1

License: (a) This work is licensed under a Creative Commons Attribution 4.0 International License.

Read Full License

Version of Record: A version of this preprint was published at BMC Family Practice on May 9th, 2020. See the published version at https://doi.org/10.1186/s12875-020-01158-1. 


\section{Abstract}

Background This study evaluated the impact of multifaceted NPS MedicineWise programs conducted in 2009 and 2015 that targeted general practitioners (GPs) to reduce unnecessary prescribing of proton pump inhibitors (PPIs). Methods Time series analyses of the dispensing rates of concessional PPI scripts between January 2006 and June 2016 using the Pharmaceutical Benefits Scheme (PBS) and Medicare Benefits Schedule (MBS) databases in Australia. Participants were GPs with dispensed PPI prescriptions to concessional patients between January 2006 and June 2016. The interventions were National NPS MedicineWise PPI educational programs in 2009 and 2015 delivered to all practising GPs in Australia. The 2015 intervention coincided with the release of Choosing Wisely Australia recommendations from the RACGP. Outcome measures included monthly dispensing rates of standard and low strength PPIs prescribed by GPs among concessional patients in Australia. Results Following the 2009 NPS MedicineWise program we observed a $6.7 \%$ reduction in the expected dispensing rate of standard strength PPIs among concessional patients between January 2006 and March 2015, and a total 8.6\% reduction by June 2016 following the launch of the 2015 program. We observed a significant increase of $5.6 \%$ in the expected dispensing rate of low strength PPIs among concessional patients between April 2009 and March 2015, and no significant change in trend following the 2015 program. Conclusions The NPS MedicineWise programs were associated with reductions in the dispensing rate of standard strength PPIs, and with an increase in the dispensing rate of low-strength PPIs by June 2016. Although the rate of high-strength PPI prescribing is declining, these formulations still constitute the majority of PPI use in Australia. Regular interventions to sustain and improve PPI management by GPs may be warranted.

\section{Background}

Proton Pump Inhibitors (PPIs) are used to treat symptoms of gastro-oesophageal reflux disease (GORD), peptic ulcer disease, functional dyspepsia, Barrett's oesophagus, and oesophagitis (1-3). Clinical guidelines recommend that patients receive PPI treatment for 4 to 8 weeks on standard strength PPIs and step down to a lower strength or discontinue treatment thereafter, while a limited number of indications may require ongoing, maintenance treatment $(1,4)$. Despite these recommendations there is growing evidence that PPIs are being inappropriately prescribed in contemporary practice leading to overuse in both primary care and hospital settings (5-7). In Australia PPI usage increased from 44.0 defined daily doses (DDDs) per 1000 population in 2000 to 77.5 in 2015 (8), and "standard" strength formulations-the highest strengths of each PPI medicine available (Table 1)-constitute the majority of dispensed PPI treatment (9).

While PPIs are generally well tolerated, their use has been linked to an increased risk of a number of adverse outcomes (10-15). In the past decade, initiatives such as educational programs conducted by NPS MedicineWise and recommendations by Choosing Wisely Australia $(1,16,17)$ have been launched with the aim of improving the quality use of PPI medicines and reducing the risk of PPI-related adverse outcomes. The Choosing Wisely program is a physician-led initiative that aims to encourage discussions between physicians and patients around practices that provide little value or may unnecessarily harm 
patients $(16,18)$. In Australia, the program is facilitated by NPS MedicineWise, a national, not-for-profit organisation seeking to improve the quality use of medicines and medical tests in Australia through educational campaigns that target general practitioners (GPs), pharmacists, other health professionals, and consumers.

In 2009 and 2015, NPS MedicineWise launched two multifaceted programs targeting general practitioners (GPs) with messages about the quality prescribing of PPIs $(19,20)$. Among other aspects, these programs promoted the appropriate use of step-down PPI treatment approaches. The aim of this study was to evaluate the impact of these interventions on dispensing rates of low and standard strength PPIs across Australia.

\section{Methods}

Description of the Interventions The April 2009 and April 2015 NPS MedicineWise PPI programs sought to provide and reinforce evidence-based recommendations to guide GPs in the appropriate primary care management of GORD, and to promote dialogue between GPs and patients about the relative benefits, risks, harms and costs of treatments. The programs included a review of recent safety updates from the Australian Therapeutic Goods Administration and tools for GPs to facilitate step-down PPI therapy in patients whose reflux symptoms were well-controlled (19). GPs were further provided with feedback detailing their prescribing behaviours during the calendar years preceding each intervention (2008 and 2014). This feedback was provided in the form of an individualised report sent to all registered and practicing GPs in Australia. The report used national medicines dispensing records from the Pharmaceutical Benefits Scheme (PBS) to summarise all PPI medicines prescribed by each GP, and how their prescribing of these distinct medicines compared to that of all other GPs in Australia, for each medicine. The report also highlighted how their prescribing aligned with best practice recommendations. This feedback was provided to approximately 20,000 GPs in 2009 and 24,000 GPs in 2015 and included the number of PPI prescriptions dispensed each month, the strengths of the prescribed PPIs, the cost of the prescribed PPIs, and the number of their patients estimated to be receiving long-term PPI treatment (defined as patients with six or more PPI dispensings). In 2009 and 2015, these feedback reports were followed by a clinical audit and a case study for a sample of GPs receiving the reports. See Table 2 for the details and the reach of each of these aspects of the interventions. The key recommendations to GPs in the NPS MedicineWise programs in 2009 and 2015 were: Review all patients currently being treated with PPIs Confirm that the indication for treatment remains; evaluate whether or not the strength and frequency of PPI dosing can be reduced; and evaluate whether or not PPI therapy can be discontinued Encourage lifestyle modifications and review the concomitant use of medicines that may exacerbate symptoms Decrease PPI treatment to low strength or intermittent, symptom-driven therapy (pro re nata) once symptoms are controlled Always discuss the expected duration of treatment and have a plan for stepping down or discontinuing treatment when PPI treatment is initiated. Setting and Data Australia maintains a universal healthcare system entitling all citizens and permanent residents to subsidised medicines through the PBS and subsidised outpatient medical services through the Medicare Benefits Schedule. The Australian Government Department of Human Services (DHS)-administering body for the 
PBS and MBS-supplied summary data of monthly dispensing records of PPI medicines subsidised through the PBS, prescribed by a GP, for each GP; and monthly records of every medical service (21) billed to the government by each GP in Australia from January 2006 through June 2016. GPs comprised registered general practitioners, trainees and non-vocationally recognised doctors. The DHS generated a unique identifier code for each GP in the data and this code allowed us to link dispensing and services data. PBS data capture prescription medicine dispensing that has resulted in a subsidy paid by the PBS; the data do not capture dispensing of medicines priced below the PBS co-payment threshold or medicines dispensed privately. The price of many PPI medicines is below the general PBS co-payment threshold (range: $\$ 29.50$ - $\$ 38.30$ between 2006 and 2016) but above the concessional PBS co-payment threshold (range: $\$ 4.70$ - $\$ 6.20$ between 2006 and 2016). To ensure complete ascertainment of PPIs dispensed during the study period, we restricted our analyses to concessional beneficiaries (22). Measures As actual GP prescribing records are not available in our dispensing data, we examined the impact of the NPS MedicineWise interventions on the rate of dispensed PPI prescriptions issued by GPs as a surrogate measure of GP prescribing. Our outcome measures were constructed as the monthly number of standard and low strength PPIs (Table 1) dispensed through the PBS (numerators) per 1,000 reimbursable GP consultations (denominator) $(23,24)$. In the case of esomeprazole, the only PBSsubsidised strengths available in Australia are classified as "high" and "standard" strength. For the purpose of our analyses, we considered dispensings of the highest available strength esomeprazole ("high") as a standard strength PPI, and the lowest available strength esomeprazole ("standard") as a low strength PPI. Statistical analysis We used time series intervention models with an autoregressive residual process to analyse the dispensing rate of standard and low strength PPIs separately. We adjusted our data seasonally to account for the well-known "stockpiling" phenomenon that results in increased dispensing of many medicines subsidised through the PBS towards the end of each calendar year, and subsequent reduced dispensing during the following January and February (22). We conducted our analyses using the seasonally adjusted data but added the unadjusted data series to the final graphic presentations. We used two separate change-in-trend variables to represent the NPS MedicineWise interventions in 2009 and 2015. We hypothesised that the impact of the 2009 intervention would diminish with time and included a decay term for the 2009 program in our models to test this hypothesis. If the estimate of a decay effect was not significant, we set the decay parameter to zero and re-estimated the model. We did not include a decay term for the 2015 intervention as fewer time points were available between April 2015 and the end of our data series (June 2016). We used a linear underlying trend to fit the dispensings rate for standard strength PPIs and a square root of linear trend to represent the underlying trend in the dispensing rate for low strength PPIs. We used the statistical package, mgcv, to perform seasonal adjustment of the data series (25) and estimated the intervention models using generalised non-linear least squares with the package nlme (26). We performed all analyses in R v3.3.3 (27) and used a p-value of less than 0.05 to indicate statistical significance.

\section{Results}


We found that the 2009 NPS MedicineWise program was associated with a statistically significant reduction in the dispensing rate of standard strength PPIs $(p<0.0001$; Figure 1$)$ and a significant increase in the rate of low strength PPI dispensing ( $<<0.0001$; Figure 2). The 2015 NPS MedicineWise program was associated with a statistically significant reduction in the dispensing rate of standard strength PPIs $(p<0.0001)$ but no change in the rate of low strength PPI dispensing (Table 3$)$.

Compared to the expected rate of standard strength PPI dispensing without the interventions, we observed a $6.7 \%$ reduction in the rate of standard strength PPI dispensing from April 2009 until March 2015 , with a total $8.6 \%$ reduction by June 2016 (Figure 1). The estimated reduction in dispensing of standard strength PPIs was 5.5/1,000 GP consultations per month following the 2009 program, and $14.9 / 1,000$ GP consultations per month following the 2015 program. We did not observe evidence of a decay effect associated with the 2009 intervention (Table 3).

Compared to the expected rate of low strength PPI dispensing without the interventions, we observed a 5.6\% increase in the dispensing rate by March 2015 (Figure 2). By June 2016, the overall increase in the dispensing rate of low strength PPIs was $5.0 \%$. The estimated increase in the dispensing rate of low strength PPIs was 1.5/1,000 GP consultations per month following the 2009 program. We observed a slight, but non-significant increase in the rate of low strength PPI dispensing following the 2015 intervention (Table 3).

\section{Discussion}

Our study demonstrated significant changes in the rates of PPI dispensing, as a surrogate measure of GP prescribing, following two targeted interventions, using national, whole-of-population dispensing data. We found that rates of standard strength PPI dispensing declined following the interventions, while dispensing rates for low strength PPIs increased following the 2009 intervention but not the 2015 intervention. These findings suggest that the NPS MedicineWise programs were effective in improving quality PPI prescribing by GPs in Australia.

Our results are consistent with recent studies of Australian veterans, which found that interventions conducted in 2004, 2006 and 2009 by NPS MedicineWise and the Veterans' MATES program in 2006 and 2012 resulted in a $20.9 \%$ relative decrease in overall PPI dispensing and a $42.2 \%$ relative increase in low strength PPI dispensing 12 months after the final intervention in the veteran population (28). Medicines dispensed to Australian veterans are subsidised through the Repatriation Pharmaceutical Benefits Scheme (RPBS), a funding body distinct to the PBS. Our data did not include RPBS dispensing records and our findings suggest that GPs may have applied new knowledge resulting from these programs in treating both their veteran and other community patients.

We estimated that the 2015 NPS MedicineWise program was associated with a $3.0 \%$ decrease in the standard strength PPI dispensing rate in the first 15 months following the intervention. This was greater than the $1.6 \%$ decrease during the 15 months following the 2009 intervention and the larger drop may be related to the Choosing Wisely Australia PPI campaign launched during the same month as the NPS 
MedicineWise 2015 intervention. The Choosing Wisely campaign was aimed at beginning a conversation between GPs and patients around the long-term use of PPIs; with Choosing Wisely members recommending regular attempts at lower-strength prescribing or cessation of PPI therapy in patients with uncomplicated disease (17). Although the impacts of the Choosing Wisely campaign and NPS MedicineWise program could not be separated, it is possible that each reinforced the messages of the other, resulting in a further reduction in the observed use of standard strength PPIs.

Our study highlights the benefits of engaging with practitioners to improve the quality use of medicines. The goal of Choosing Wisely is to start discussions between physicians and patients around specific therapeutic practices. NPS MedicineWise actively engages general practitioners and consumers in educational activities aimed to improve the quality use of medicines through seeking behaviour change (29). The collaboration of these groups suggests opportunities for additional quality-use-of-medicines interventions, as well as on-going PPI education.

\section{Strengths \& Limitations}

Our study examined PPIs dispensed to concessional beneficiaries. The cost of all PPI medicines subsidised by the PBS is above the concessional co-payment threshold and, therefore, we have a complete ascertainment of PPI dispensing for these beneficiaries. The DHS began collecting dispensing data for medicines where cost was below the co-payment threshold for general beneficiaries from April 2012 and based on dispensing data from that time it has been estimated that approximately $70 \%$ of PPIs prescribed by GPs were dispensed to concessional beneficiaries. We expect the intervention impacts we observed in concessional beneficiaries would be similar among general beneficiaries as we do not expect the treatment of GORD with PPIs to differ between the two subsidy groups.

Our study is observational, and causality cannot be confirmed. Furthermore, standard strength esomeprazole received approval for over-the-counter (OTC) marketing in Australia in February 2016, and some of the reduction in standard strength PPI dispensing we observed following the April 2015 intervention could have been due to patients switching from prescription to OTC access for this medicine. However, the OTC formulation contained just a 7-day supply and the cost was above that of the concessional beneficiary co-payment in $2016(\$ 6.20)$. We believe it is unlikely that many concessional beneficiaries would have switched to an OTC formulation. Given the timing of the intervention programs, and that we did not identify any other potential confounding events following the 2009 and the 2015 programs that might explain the changes we observed, we believe that the change in the rate of PPI dispensing is attributable to the NPS MedicineWise programs and the Choosing Wisely RACGP recommendation.

PBS data are maintained for the purpose of providing reimbursement to patients and pharmacies, and clinical information such as diagnoses and treatment indications are not captured. Although the programs we evaluated were aimed at improving the quality use of PPIs, we are unable to assess the 
appropriateness of prescribing using these data. Similarly, our prescriber-level PBS data do not allow us to evaluate the rates at which individual patients switched between PPI treatment strengths or ceased PPI therapy.

The strengths of our study include its longitudinal and whole-of-population capture-our data comprised dispensing records for all medicines prescribed by every general practitioner in Australia, followed from 2006 through June 2016. These data allowed for robust estimates of trends over time in the exact population targeted by the interventions.

\section{Conclusions}

The NPS MedicineWise programs were effective in changing Australian general practitioner's prescribing of PPI medicines. These programs were complemented by a Choosing Wisely recommendation produced by RACGP which may have resulted in more efficacious interventions aimed at improving quality prescribing. Regular educational programs targeting the prescribing of PPIs may ensure that quality prescribing practices are continued.

\section{Abbreviations}

DDDs - defined daily doses

GP - general practitioner

MBS - Medicare Benefits Schedule

OTC- over-the-counter

PBS - Pharmaceutical Benefits Scheme

PPI - proton pump inhibitors

RACGP - Royal Australian College of General Practice

\section{Declarations}

This research was funded by the Australian Government Department of Health through NPS MedicineWise. We acknowledge all staff at NPS MedicineWise who contributed and continue to contribute to the PPI programs. We acknowledge the Australian Government Department of Human Services for provision of the data used in this study.

Declaration: 
Ethics approval and consent to participate: All data is deidentified and there was no further requirement for ethics approval.

Consent for publication:

NPS MedicineWise and the Department of Human Services approved this manuscript before submission.

Availability of data and material:

PBS and MBS data is available online - see the Australian Department of Health http://www.pbs.gov.au/info/statistics/expenditure-prescriptions/pbs-expenditure-and-prescriptions http://medicarestatistics.humanservices.gov.au/statistics/mbs_item.jsp

Competing interests:

The authors declare that they have no competing interests.

Funding:

Funding was provided the Australian Department of Health to NPS MedicineWise.

Author's contributions:

JW analysed and interpreted the PBS and MBS data and contributed in writing the manuscript

SD contributed to the background information and the writing of the manuscript

Zain Elgebaly contributed to the intervention design and the writing of the manuscript

Suzanne Blogg contributed to the writing of the manuscript

Aine Heaney contributed to the manuscript

Yien Soo contributed to the intervention design and the writing of the manuscript

Benjamin Daniels contributed to the analysis and the writing of the manuscript

Lynn Weekes contributed to the writing of the manuscript 
All authors read and approved the final manuscript.

Acknowledgements:

Not applicable

\section{References}

1. Choosing Wisely Australia. The Royal Australian College of General Practitioners: tests, treatments and procedures clinicians and consumers should question. Choosing Wisely Australia 2015 [Available from: http://www.choosingwisely.org.au/recommendations/racgp.

2. Cancer Council Australia. Clinical practice guidelines for the diagnosis and management of Barrett's Oesophagus and Early Oesophageal Adenocarcinoma 2014 [Available from: https://wiki.cancer.org.au/australia/Guidelines:Barrett\%27s.

3. Gastrointestinal Expert Group. Therapeutic guidelines: gastrointestinal - disorders of the oesophagus Melbourne: Therapeutic Guidelines Limited; 2014 [Available from: https://tgldcdp.tg.org.au/viewTopic?topicfile=disorders-oesophagus - toc_d1e47.

4. Yadlapati R, Kahrilas PJ. When is proton pump inhibitor use appropriate? BMC Medicine. 2017;15(1):36.

5. Ahrens D, Behrens G, Himmel W, Kochen MM, Chenot J-F. Appropriateness of proton pump inhibitor recommendations at hospital discharge and continuation in primary care. International Journal of Clinical Practice. 2012;66(8):767-73.

6. Batuwitage BT, Kingham JGC, Morgan NE, Bartlett RL. Inappropriate prescribing of proton pump inhibitors in primary care. Postgraduate medical journal. 2007;83(975):66-8.

7. Kelly OB, Dillane C, Patchett SE, Harewood GC, Murray FE. The Inappropriate Prescription of Oral Proton Pump Inhibitors in the Hospital Setting: A Prospective Cross-Sectional Study. Digestive Diseases and Sciences. 2015;60(8):2280-6.

8. Organisation for Economic Co-operation and Development. OECD.Stat 2018 [Available from: https://stats.oecd.org/Index.aspx?DataSetCode=HEALTH_PHMC.

9. Hollingworth S, Duncan EL, Martin JH. Marked increase in proton pump inhibitors use in Australia. Pharmacoepidemiology and Drug Safety. 2010;19(10):1019-24.

10. Giuliano C, Wilhelm SM, Kale-Pradhan PB. Are proton pump inhibitors associated with the development of community-acquired pneumonia? A meta-analysis. Expert Review of Clinical Pharmacology. 2012;5(3):337-44.

11. Gracie DJ, Ford AC. The possible risks of proton pump inhibitors. The Medical journal of Australia. 2016;205(7):292-3. 
12. Roughead EE, Chan EW, Choi NK, Griffiths J, Jin XM, Lee J, et al. Proton pump inhibitors and risk of Clostridium difficile infection: a multi-country study using sequence symmetry analysis. Expert opinion on drug safety. 2016;15(12):1589-95.

13. Therapeutic Goods Administration. Proton pump inhibitors and acute interstitial nephritis Canberra: Australian Government Department of Health and Ageing; 2011. Contract No.: 6.

14. Xie Y, Bowe B, Li T, Xian H, Yan Y, Al-Aly Z. Risk of death among users of Proton Pump Inhibitors: a longitudinal observational cohort study of United States veterans. BMJ Open. 2017;7(6):e015735.

15. Yang YX, Metz DC. Safety of Proton Pump Inhibitor Exposure. Gastroenterology. 2010;139(4):111527.

16. Choosing Wisely Australia. Choosing Wisely Australia [Available from: http://www.choosingwisely.org.au/home.

17. Choosing Wisely Australia. Gastroenterological Society of Australia: tests, treatments and procedures clinicians and consumers should question 2015 [Available from: http://www.choosingwisely.org.au/recommendations/gesa.

18. Choosing Wisely. Choosing Wisely: Our Mission 2018 [Available from: https://www.choosingwisely.org/our-mission/.

19. NPS MedicineWise. Proton pump inhibitors: too much of a good thing? NPS MedicineWise 2015 [Available from: https://www.nps.org.au/medical-info/clinical-topics/news/proton-pump-inhibitorstoo-much-of-a-good-thing

20. NPS MedicineWise. National Consumer Survey: audience insights report. Sydney: National Prescribing Service Ltd; 2017.

21. Australian Government Department of Health. MBS Online 2018 [Available from: http://www.mbsonline.gov.au/internet/mbsonline/publishing.nsf/Content/Home.

22. Mellish L, Karanges EA, Litchfield MJ, Schaffer AL, Blanch B, Daniels BJ, et al. The Australian Pharmaceutical Benefits Scheme data collection: a practical guide for researchers. BMC Research Notes. 2015;8(1):634.

23. Mandryk JA, Mackson JM, Horn FE, Wutzke SE, Badcock C-A, Hyndman RJ, et al. Measuring change in prescription drug utilization in Australia. Pharmacoepidemiology and Drug Safety. 2006;15(7):47784.

24. Horn FE, Mandryk JA, Mackson JM, Wutzke SE, Weekes LM, Hyndman RJ. Measurement of changes in antihypertensive drug utilisation following primary care educational interventions. Pharmacoepidemiol Drug Saf. 2007;16(3):297-308.

25. Wood S. mgcv: Mixed GAM Computation Vehicle with Automatic Smoothness Estimation. R package version 1.8 - 22 ed2017.

26. J P, D B, S D, D S, (2017) RCT._nlme: Linear and Nonlinear Mixed Effects Models_. R package version 3.1-131 ed. 
27. Team RC. R: A language and environment for statistical computing. R Foundation for Statistical Computing, Vienna, Austria; 2017.

28. Pratt NL, Kalisch Ellett LM, Sluggett JK, Gadzhanova SV, Ramsay EN, Kerr M, et al. Use of proton pump inhibitors among older Australians: national quality improvement programmes have led to sustained practice change. International journal for quality in health care : journal of the International Society for Quality in Health Care. 2017;29(1):75-82.

29. NPS MedicineWise. About NPS MedicineWise 2018 [Available from: https://www.nps.org.au/aboutus.

\section{Tables}

Table 1. PBS item codes used in the analysis of the NPS MedicineWise programs

\begin{tabular}{|c|c|c|}
\hline Drug name & Strength & PBS item numbers \\
\hline \multirow[t]{2}{*}{ Esomeprazole } & Standard strength* & $8601 \mathrm{Q}$ \\
\hline & Low strength ${ }^{*}$ & 8600P, 8886Q \\
\hline \multirow[t]{2}{*}{ Lansoprazole } & Standard strength & 2240X, 2241Y, 8528W, 8529X, 8949B, 8950C, 9477T, 9478W \\
\hline & Low strength & 8198L, 9331D \\
\hline \multirow[t]{2}{*}{ Omeprazole } & Standard strength & 1326T, 1327W, 8331L, 8333N, 8776X, 8777Y, 9109K, 9110L \\
\hline & Low strength & 8232G, 8332M \\
\hline \multirow[t]{2}{*}{ Pantoprazole } & Standard strength & $8007 \mathrm{~K}, 8008 \mathrm{~L}, 9423 \mathrm{Y}, 9424 \mathrm{~B}$ \\
\hline & Low strength & $8399 \mathrm{C}$ \\
\hline \multirow[t]{2}{*}{ Rabeprazole } & Standard strength & $8508 \mathrm{~T}, 8509 \mathrm{~W}$ \\
\hline & Low strength & $8507 \mathrm{R}$ \\
\hline
\end{tabular}

* There is no PBS-subsidised "low" strength formulation for esomeprazole. For the purposes of our analyses, we considered dispensings of the highest available strength esomeprazole ("high"; PBS item number 8601Q) as a standard strength PPI, and the lowest available strength esomeprazole ("standard"; PBS Item numbers 8600P \& 8886Q) as a low strength PPI.

Table 2: NPS MedicineWise interventions targeting PPI prescribing in Australia 


\begin{tabular}{|l|l|l|l|l|}
\hline Intervention & Date & Details & \multicolumn{2}{|l|}{$\begin{array}{l}\text { Number GPs } \\
\text { reached }\end{array}$} \\
\cline { 4 - 6 } & & & 2009 & 2015 \\
\hline $\begin{array}{l}\text { PBS } \\
\text { feedback }\end{array}$ & $\begin{array}{l}2009 \\
\text { and } \\
2015\end{array}$ & $\begin{array}{l}\text { A personalised prescribing report was sent to registered practicing } \\
\text { GPs in Australia. The distribution of feedback reports was } \\
\text { coordinated with the Department of Human Services by using PBS } \\
\text { data. }\end{array}$ & 19,790 & 24,188 \\
\hline Case study & $\begin{array}{l}2009 \\
\text { and } \\
2015\end{array}$ & $\begin{array}{l}\text { The educational activity explored how to manage a new PPI therapy } \\
\text { according to best practice recommendations and it provided GP } \\
\text { participants with immediate information on how their prescribing } \\
\text { compared with their peers }\end{array}$ & 169 & 397 \\
\hline $\begin{array}{l}\text { Clinical } \\
\text { Audit }\end{array}$ & $\begin{array}{l}2009 \\
\text { and } \\
\text { 2015 }\end{array}$ & $\begin{array}{l}\text { GPs review their current prescribing practice for their patients } \\
\text { compared with current best practice guidelines }\end{array}$ & 1590 & 687 \\
\hline
\end{tabular}

Table 1. PBS item codes used in the analysis of the NPS MedicineWise programs

\begin{tabular}{llll} 
Drug name & Strength & PBS item numbers \\
\hline Esomeprazole & Standard strength $^{*}$ & 8601Q \\
\cline { 2 - 3 } & Low strength* & 8600P, 8886Q \\
\hline Lansoprazole & Standard strength & 2240X, 2241Y, 8528W, 8529X, 8949B, 8950C, 9477T, 9478W \\
\cline { 2 - 3 } & Low strength & 8198L, 9331D \\
\hline Omeprazole & Standard strength & 1326T, 1327W, 8331L, 8333N, 8776X, 8777Y, 9109K, 9110L \\
\cline { 2 - 3 } & Low strength & 8232G, 8332M \\
\hline Pantoprazole & Standard strength & 8007K, 8008L, 9423Y, 9424B \\
\cline { 2 - 3 } & Low strength & 8399C \\
\hline Rabeprazole & Standard strength & 8508T, 8509W \\
\cline { 2 - 3 } & Low strength & 8507R \\
\hline
\end{tabular}

* There is no PBS-subsidised "low" strength formulation for esomeprazole. For the purposes of our analyses, we considered dispensings of the highest available strength esomeprazole ("high"; PBS item number 8601Q) as a standard strength PPI, and the lowest available strength esomeprazole ("standard"; PBS Item numbers 8600P \& 8886Q) as a low strength PPI.

Table 3: Generalised linear and non-linear least square estimates of high and low strength PPIs monthly time series data 


\begin{tabular}{|c|c|c|c|c|c|c|}
\hline Variables in the Model & \multicolumn{3}{|c|}{ High Strength PPIs } & \multicolumn{3}{c|}{ Low Strength PPIs } \\
\cline { 2 - 7 } & Coefficient & $95 \%$ CI & $p$-Value & Coefficient & $95 \%$ CI & $p$-Value \\
\hline Intercept & 70.077 & $(69.09,70.06)$ & $<0.0001$ & 13.546 & $(12.98,14.17)$ & $<0.0001$ \\
\hline Trend & 0.163 & $(0.13,0.20)$ & $<0.0001$ & & & \\
\hline & & & & 1.563 & $(1.43,1.67)$ & $<0.0001$ \\
\hline NPS 2009 & -0.153 & $(-0.20,-0.11)$ & $<0.0001$ & 0.282 & $(0.22,0.36)$ & $<0.0001$ \\
\hline Decay & - & - & - & 0.047 & $(0.036,0.062)$. & $<0.0001$ \\
\hline NPS 2015 & -0.334 & $(-0.46,-0.21)$ & $<0.0001$ & 0.030 & $(-0.028,0.087)$ & 0.296 \\
\hline Auto-correlation f & -0.193 & $(-0.36,-0.015)$ & 0.0313 & - & - & - \\
\hline
\end{tabular}

\section{Figures}

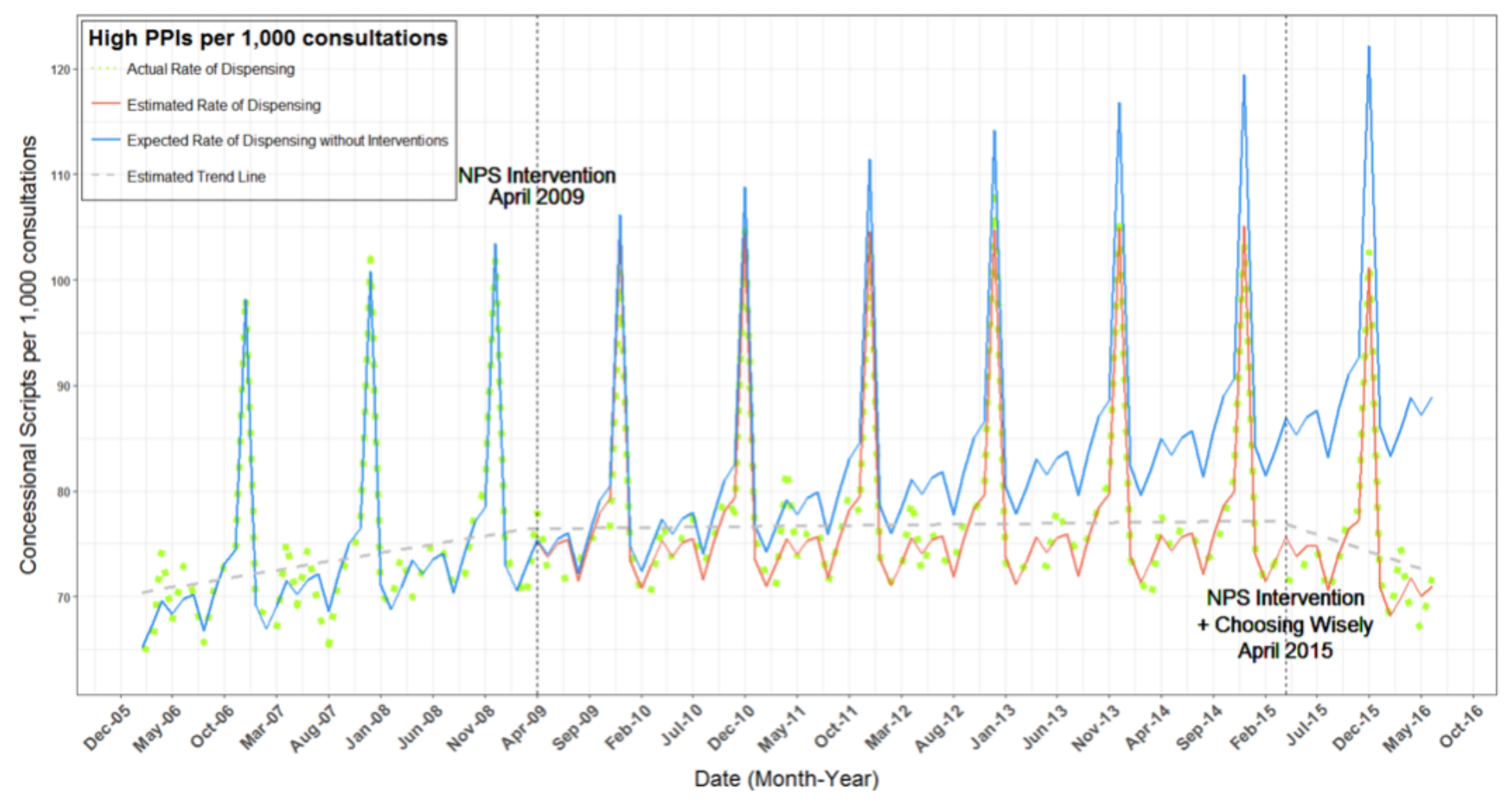

Figure 1

Fitted model for the number of concessional high strength PPIs dispensing per 1,000 consultations between Jan-2006 and Jun-2016 


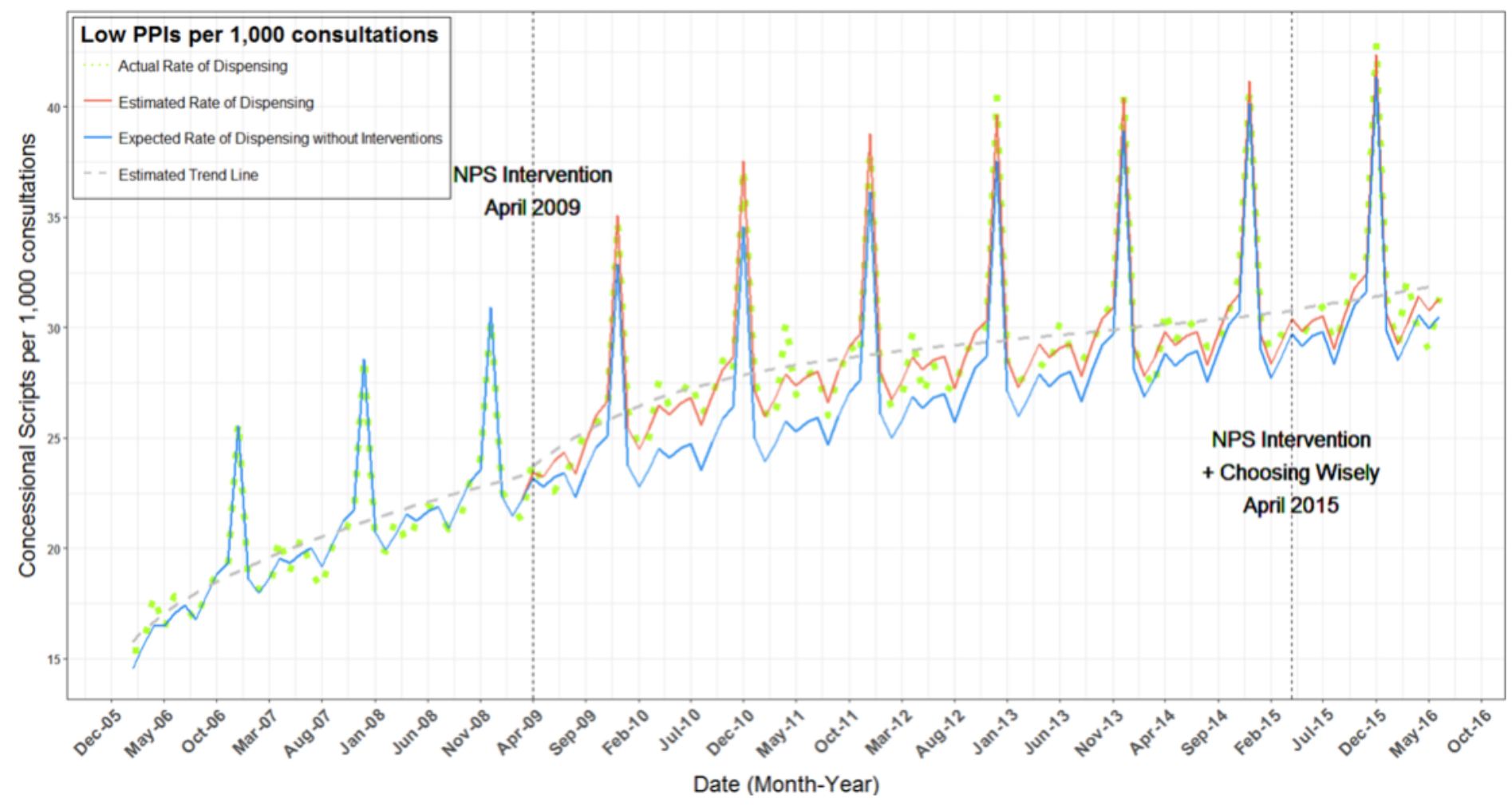

Figure 2

Fitted model for number of concessional low strength PPIs dispensing per 1,000 consultations between January 2006 and June 2016 